\title{
Clinical significance of CXCL13/CXCR5 axis in human cancers
}

\author{
Dawei Zhu ${ }^{1,2,3}$, Wenfeng Ye ${ }^{1,2,3}$, Jingting Jiang ${ }^{1,2,3}$ \\ ${ }^{1}$ Department of Tumor Biological Treatment, ${ }^{2}$ Jiangsu Engineering Research Center for Tumor Immunotherapy, ${ }^{3}$ Institute of Cell Therapy, the \\ Third Affiliated Hospital of Soochow University, Changzhou 213003, China \\ Contributions: (I) Conception and design: J Jiang, D Zhu; (II) Administrative support: J Jiang; (III) Provision of study materials or patients: None; \\ (IV) Collection and assembly of data: None; (V) Data analysis and interpretation: None; (VI) Manuscript writing: All authors; (IV) Final approval of \\ manuscript: All authors. \\ Correspondence to: Jingting Jiang, MD, PhD. Department of Tumor Biological Treatment, the Third Affiliated Hospital, Soochow University, \\ Changzhou 213003, China. Email: jiangjingting@suda.edu.cn.
}

\begin{abstract}
As a family of small cytokines, chemokines were well known to contribute to the migration of cells, especially the inflammatory cells such as chemotactic granulocytes, monocytes and macrophages, and their functions are mediated by the corresponding receptors. Both chemokines and their receptors not only play an important role in the physiopathological process of inflammation and anaphylaxis, but also are involved in tumor growth and metastasis. For example, numerous studies have shown that chemokines can regulate the infiltration of leukocytes in tumor tissues, the tumor-associated angiogenesis, and the hostspecific immune responses against tumors. Moreover, chemokines could also contribute to the regulation of cancer cell proliferation and motility through autocrine or paracrine processes. In the present review, we summarized the clinical significance and the function of CXCL13/CXCR5 axis in human cancers.
\end{abstract}

Keywords: Chemokine; CXCL13; CXCR5; cancer progression

Submitted Jul 25, 2018. Accepted for publication Nov 05, 2018.

doi: $10.21037 /$ tcr.2018.11.26

View this article at: http://dx.doi.org/10.21037/tcr.2018.11.26

Chemokines and their receptors are important molecules mediating leukocyte migration and infiltration. Abnormal expressions of tumor-associated chemotactic factors in tumor tissues are significantly related to cancer progression, lymphocyte infiltration and specific antitumor immune responses. Recently, many studies have shown that the chemokine CXCL13 and its receptor CXCR5 were abnormally expressed in cancer tissues and contributed essentially to cancer progression (1). In the present review, we summarized the expression pattern and clinical significance of CXCL13/CXCR5 axis in human cancers.

\section{Chemokines and their receptors}

As small molecule peptides with a molecular weight of $8-10 \mathrm{kD}$, chemokines can specifically activate and recruit white blood cells $(2,3)$. The amino acid composition of chemokines has a specific cysteine (Cys) motif. According to the characteristics of the conservative arrangement of Cys motifs, the chemokines can be mainly categorized into four subgroups (CXC, CC, C and CX3C) (4,5). Up to date, most of the chemokines belong to the $\mathrm{CC}$ and CXC subgroups, while only two $\mathrm{C}$ molecules and one $\mathrm{CX} 3 \mathrm{C}$ molecule have been reported. Chemokine receptors are a group of $\mathrm{G}$ protein-coupled trans-membranous receptors that mediate the ability of chemokines and contain seven transmembrane domains (6). Chemokine receptors could be found in various immune cells, endothelial cells and neuronal cells, and each receptor has a retention region that binds to the corresponding chemokine ligand (7). The chemokines can participate in the physio-pathological process of infectious and metamorphic diseases, and chemokines as well as their receptors can also promote angiogenesis, tumor cell proliferation, survival and proliferation, involving in the process of organ-specific metastasis of malignant tumors. 
Table 1 CXCL13/CXCR5 axis in human cancers

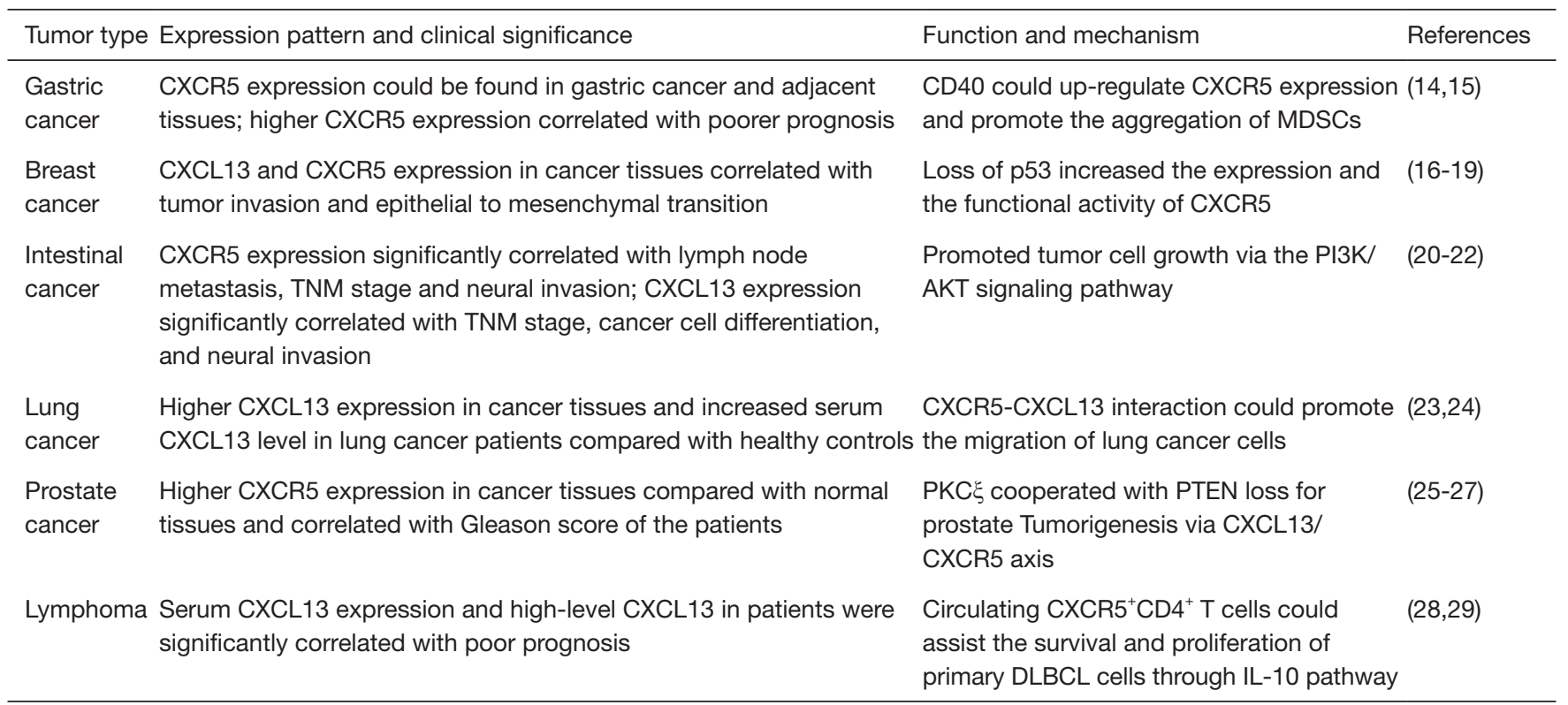

\section{Chemokine CXCL13 and its receptor CXCR5}

CXCR5, also known as Burkitt's lymphoma receptor 1 (BLR-1), is a member of the chemokine CXC receptor family, which is mainly distributed in peripheral mature $\mathrm{B}$ cells as well as in a small number of $\mathrm{CD}^{+}$or $\mathrm{CD}^{+} \mathrm{T}$ cells and the skin-derived migratory dendritic cells (8). CXCR5 has seven transmembrane domains, and it couples with GTP2 protein to form a transmembrane receptor. CXCR5 is an important molecule that enables B lymphocytes to home to lymph node follicles and to promote lymphoid follicular formation and lymph node development. CXCL13, also known as B cell chemokine 1 (BLC1), is a ligand of CXCR5 and a member of chemokine CXC family. The coding gene of CXCL13 is located in $4 \mathrm{q} 21$. Generally, CXCL13 is secreted by stromal cells in the B cell region of the secondary lymphoid tissue and expressed on the membrane of the follicular dendritic cell. CXCL13 can recruit $\mathrm{B}$ cells and directly regulate the maturation and development of B cells in vivo, and can participate in the transmission of immune system signals $(9,10)$. CXCL13/CXCR5 axis contributes to the homing process of circulating naive $\mathrm{B}$ lymphocytes to lymph nodes, promotes the construction and the development of secondary lymphoid tissue, and facilitates the B cells to differentiate into functional B Cells. Recent studies have also shown that CXCL13 and its receptor CXCR5 not only are involved in maintaining the homeostasis of tissue environment, but also participate in the occurrence and treatment of autoimmune diseases as well as the repair of trauma (11-13). Moreover, many studies also showed that the CXCL13/CXCR5 axis played a fundamental role in regulating tumor occurrence and development, and herein we reviewed these studies and summarized in Table 1.

\section{Studies on CXCL13/CXCR5 axis in human cancers}

\section{CXCL13/CXCR5 and gastric cancer}

Sun et al. (15) have shown that CXCR5 is highly expressed in human gastric cancer cell lines BGC-823, SGC-7901 and HGC-27. The tissue microarray and immunohistochemistry have also shown that CXCR5 is highly expressed in human gastric cancer tissues, and its expression level is significantly correlated to tumor size and overall survival rate of the patients, therefore the examination of CXCR5 may have a great importance in the prognostic prediction of gastric cancer. Ding et al. (14) also confirmed that CXCL13/ CXCR5 axis played an important role in the migration of $\mathrm{CD} 40^{+} \mathrm{MDSC}$ to gastric cancer tissues, indicating that the expression of CD40 can up-regulate the CXCR5 expression and promote the migration and recruitment of MDSCs to cancer tissues. Wei et al. (30) also showed that higher CXCL13 expression level was associated with larger tumor size and poorer prognoses of gastric cancer patients, and could serve as a predictive biomarker of response to 
postoperative adjuvant chemotherapy in these patients.

\section{CXCL13/CXCR5 and breast cancer}

Chen et al. (16) have demonstrated that there is a significant correlation between the CXCL13 expression level and unfavorable clinical features in patients with breast cancer. Moreover, higher CXCL13 expression could also be found in breast cancer tissues, and is significantly related to the status of lymph node metastasis, estrogen receptor status and other prognostic factors (6,31). Razis et al. (17) also examined the expression of CXCL13/CXCR5 in earlystage breast cancer, and the results showed that the higher CXCR5 expression as well as CXCL13 in breast cancer tissues are significantly correlated with the lower estrogen levels, poor grade and axillary lymph node metastases. Biswas et al. (18) demonstrated that the co-expression of CXCL13 and CXCR5 is significantly correlated to the status of lymph node metastasis in breast cancer patients. And in breast cancer cell lines, the over-expression of CXCL13 could promote the epithelial-mesenchymal transition (32). Mitkin et al. (19) have found that the expression of $\mathrm{p} 53$ in breast cancer cell line MCF-7 is negatively correlated with CXCR5 expression level. Furthermore, the mechanic studies have shown that CXCR5 over-expression can lead to the survival of abnormal cells in breast cancer when the function of $\mathrm{p} 53$ is missing.

\section{CXCL13/CXCR5 and colorectal cancer}

Xing et al. (22) demonstrated that the $\mathrm{CXCR}^{+} \mathrm{CD}^{+} \mathrm{T}$ cells are significantly increased in colorectal cancer tissues compared with the adjacent normal tissues, but the effector function of these $\mathrm{CXCR} 5^{+} \mathrm{CD} 8^{+} \mathrm{T}$ cells is not as good as $\mathrm{CXCR}^{-} \mathrm{CD} 8^{+} \mathrm{T}$ cells. When the $\mathrm{CXCR}^{+} \mathrm{CD}^{+} \mathrm{T}$ cells are stimulated by PMA and ionomycin, the effector function of $\mathrm{CXCR}^{+} \mathrm{CD}^{+} \mathrm{T}$ cells is significantly enhanced, indicating that $\mathrm{CXCR}^{+} \mathrm{CD}^{+} \mathrm{T}$ cells are an important subpopulation of $\mathrm{CD}^{+} \mathrm{T}$ cells infiltrating in the colon cancer tissues, and have the potential of anti-tumor immunity. Qi et al. (20) detected 144 paraffin-embedded samples of advanced colorectal cancer by using immunohistochemistry, and they have found that the expression of CXCL13 in advanced colorectal cancer tissues is significantly different from that of the normal tissues. They also confirmed that the increased protein expression levels of CXCL13 and CXCR5 are significantly correlated with the status of lymph node metastasis, distant metastasis, tumor stage and recurrence.
Zhu et al. (21) have shown that CXCL13 could promote growth, migration and invasion of colon cancer cells. CXCL13/CXCR5 axis may also promote the growth of cancer cells through the PI3K/AKT signal pathway, suggesting that CXCL13 is a potential biological marker for the detection and therapeutic treatment of colon cancer.

\section{CXCL13/CXCR5 and lung cancer}

Wang et al. found that CXCL13 is over-expressed in $62 \%$ of smokers and $45 \%$ of non-smokers in lung cancer patients (23). Mouse experiments also confirmed that the key carcinogen benzopyrene could induce the CXCL13 expression in lung epithelial cells, indicating that the important role of CXCL13/CXCR5 in PAH-induced lung cancer (23). Singh et al. (24) found that CXCR5 expression in lung cancer tissues (both squamous cell carcinoma and adenocarcinoma tissues) is significantly higher than that in adjacent normal tissues, they also showed that nuclear and membrane CXCR5 expression were higher in adenocarcinoma tissues in contrast to squamous cell carcinoma tissues. Moreover, serum CXCL13 levels in lung cancer patients were significantly higher than that in healthy controls (24). Thus all these data suggest that the CXCL13/CXCR5 axis can affect the progression of lung cancer, and both CXCL13 and CXCR5 can be used as biomarkers for lung cancer.

\section{CXCL13/CXCR5 and prostate cancer}

Singh et al. (27) reported that CXCR5 expression in prostate cancer tissues was significantly higher than that in normal tissues, and the increased intensity of CXCR5 staining significantly correlated with Gleason score of the patients. Moreover, CXCR5 expression level in prostate cancer cell lines was higher than that in normal prostatic epithelial cells, and when the prostate cancer cells treated by using CXCL13, the expression levels of collagenase-1 or matrix metalloproteinase-1 (MMP-1), collagenase-3 (MMP-13), stromelysin-1 (MMP-3), stromelysin-2 (MMP-10) and stromelysin-3 (MMP-11) were significantly increased (27). El-Haibi et al. (33) reported that CXCL13 could promote the invasion of prostate cancer cell via Akt and ERK1/2 activation and suggested a new role for DOCK 2 in proliferation of hormone-refractory CXCR5-positive prostate cancer cells. Moreover, the over-expression of protein kinase $\mathrm{C}$ epsilon and PTEN loss could individually and synergistically up-regulate the production of the 


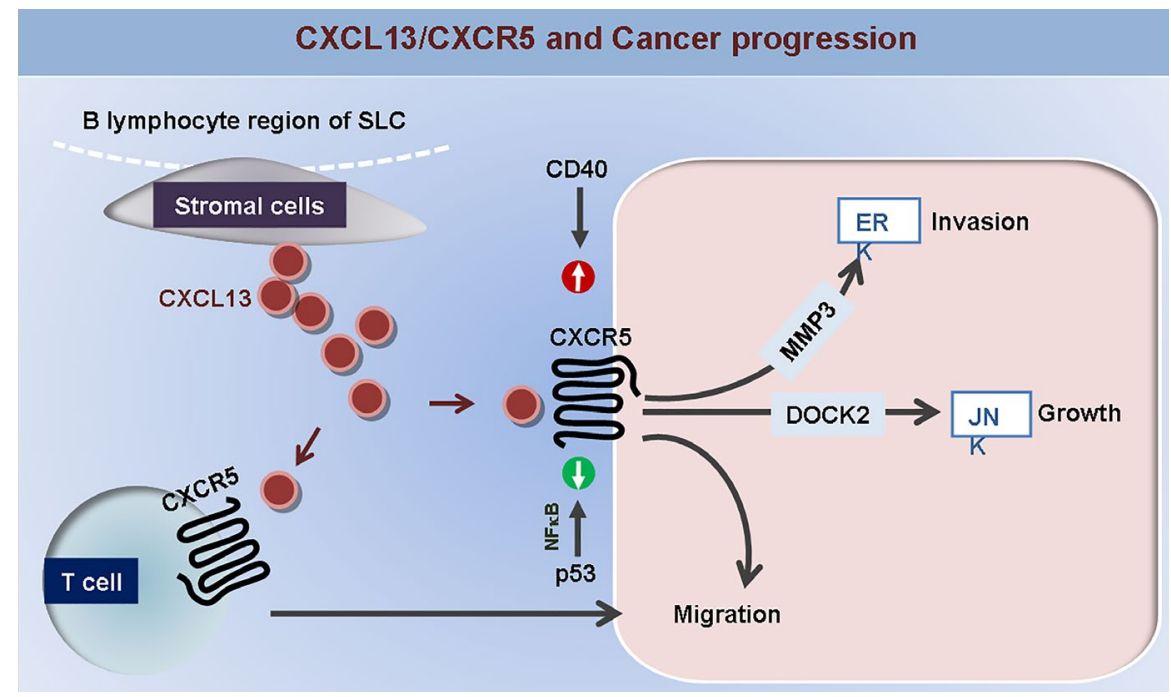

Figure 1 Graphic description of CXCL13/CXCR5 axis in cancer progression.

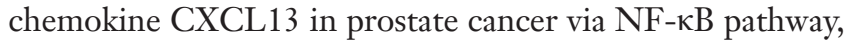
and the disruption of CXCL13/CXCR5 axis in prostate cancer cells could significantly impaired the migratory and tumorigenic properties (26).

\section{CXCL13/CXCR5 and lymphoma}

Cha et al. (29) investigated the pathogenesis of diffuse large B-cell lymphoma, and found that the peripheral CXCR $5^{+} \mathrm{CD} 4^{+} \mathrm{T}$ cells could promote tumor cell proliferation by inhibiting lymphoma cell apoptosis. Moreover, $\mathrm{CXCR}^{+} \mathrm{CD}^{+} \mathrm{T}$ cells could promote the tumorigenesis via reducing cytokine IL-12 and enhancing IL-10 secretion, indicating that CXCR5 signaling plays an important role in tumor development in this malignancy (29). Kim et al. (28) examined the serum level of CXCL13 in patients with extranodal NK/T-cell lymphoma and analyzed its correlation with the Glasgow Prognostic Score as well as its prognostic significance, and the increased serum CXCL13 level of the patients is significantly correlated with the poorer PFS and higher GPS, larger tumor burden and poorer prognosis. Therefore, the serum CXCL13 level can be used as the prognostic indicator and a potential therapeutic target for these patients.

\section{CXCL13/CXCR5 and other tumors}

Sambandam et al. (34) reported that the c-Myc activation through CXCL13/CXCR5 axis signal pathway could stimulate the expression of RANKL in stromal or preosteoblast cells, which implicated that CXCL13 could serve as a potential therapeutic target to prevent invasion of bone/osteolysis in oral squamous cell carcinoma. Duan et al. (35) have conducted a 5 -year follow-up of patients with hepatocellular carcinoma and found that the serum level of CXCL13 was significantly increased in cancer patients compared with healthy controls, and the immunohistochemical staining results also showed that the percentage of $\mathrm{CXCR}^{+}$or $\mathrm{CXCL} 13^{+}$cells in poorly differentiated patients was significantly higher than that in well-differentiated patients, and thus CXCL13 could be served as a potential biomarker for the recurrence in patients with liver cancer after hepatectomy. Del Grosso et al. (36) found that the malignant neuroblastoma cells could express CXCR5 and the Schwanno blast cells could express CXCL13, and the CXCL13 produced by stromal cells may contribute to the generation of an environment in which the malignant neuroblasts were retained.

\section{Outlook}

Numerous studies confirmed that the abnormal expression of CXCL13/CXCR5 could be found in human cancer tissues, and CXCL13/CXCR5 axis plays an important role in the regulation of cancer cell proliferation, migration and metastasis (Figure 1), and thus contributes to the cancer progression and the prognostic prediction of cancer patients. In future, the validation of CXCR5 and CXCL13 
as useful biomarkers for cancer diagnosis and prevention, and the potential value of clinical application of CXCL13/ CXCR5 axis blockade as an effective therapy against human cancers still merit further investigation.

\section{Acknowledgments}

Funding: This work was supported by the National Key R\&D Program (No. 2018YFC1313400), the National Science and Technology Supporting Program (No. 2015BAI12B12), the Joint Research Fund for Overseas Chinese, Hong Kong and Macao Scholars (No. 31729001), the National Natural Science Foundation of China (No. 31570877, No. 31570908), the Key R\&D Project of Science and Technology Department of Jiangsu Province (No. BE2018645).

\section{Footnote}

Conflicts of Interest: All authors have completed the ICMJE uniform disclosure form (available at http://dx.doi. org/10.21037/tcr.2018.11.26). The authors have no conflicts of interest to declare.

Ethical Statement: The authors are accountable for all aspects of the work in ensuring that questions related to the accuracy or integrity of any part of the work are appropriately investigated and resolved.

Open Access Statement: This is an Open Access article distributed in accordance with the Creative Commons Attribution-NonCommercial-NoDerivs 4.0 International License (CC BY-NC-ND 4.0), which permits the noncommercial replication and distribution of the article with the strict proviso that no changes or edits are made and the original work is properly cited (including links to both the formal publication through the relevant DOI and the license). See: https://creativecommons.org/licenses/by-nc-nd/4.0/.

\section{References}

1. Jacquelot N, Enot DP, Flament C, et al. Chemokine receptor patterns in lymphocytes mirror metastatic spreading in melanoma. J Clin Invest 2016;126:921-37.

2. Franciszkiewicz K, Boissonnas A, Boutet M, et al. Role of chemokines and chemokine receptors in shaping the effector phase of the antitumor immune response. Cancer Res 2012;72:6325-32.
3. Keeley EC, Mehrad B, Strieter RM. Chemokines as mediators of tumor angiogenesis and neovascularization. Exp Cell Res 2011;317:685-90.

4. Rainczuk A, Rao J, Gathercole J, et al. The emerging role of CXC chemokines in epithelial ovarian cancer. Reproduction 2012;144:303-17.

5. Periyasamy-Thandavan S, Herberg S, Arounleut P, et al. Caloric restriction and the adipokine leptin alter the SDF-1 signaling axis in bone marrow and in bone marrow derived mesenchymal stem cells. Mol Cell Endocrinol 2015;410:64-72.

6. Panse J, Friedrichs K, Marx A, et al. Chemokine CXCL13 is overexpressed in the tumour tissue and in the peripheral blood of breast cancer patients. Br J Cancer 2008;99:930-8.

7. Horuk R. Chemokine receptor antagonists: overcoming developmental hurdles. Nat Rev Drug Discov 2009;8:23-33.

8. Burkle A, Niedermeier M, Schmitt-Graff A, et al. Overexpression of the CXCR5 chemokine receptor, and its ligand, CXCL13 in B-cell chronic lymphocytic leukemia. Blood 2007;110:3316-25.

9. Ezzat M, El-Gammasy T, Shaheen K, et al. Elevated production of serum B-cell-attracting chemokine-1 (BCA1/CXCL13) is correlated with childhood-onset lupus disease activity, severity, and renal involvement. Lupus 2011;20:845-54.

10. Schiffer L, Worthmann K, Haller H, et al. CXCL13 as a new biomarker of systemic lupus erythematosus and lupus nephritis - from bench to bedside? Clin Exp Immunol 2015;179:85-9.

11. Klimatcheva E, Pandina T, Reilly C, et al. CXCL13 antibody for the treatment of autoimmune disorders. BMC Immunol 2015;16:6.

12. Worthmann K, Gueler F, von Vietinghoff S, et al. Pathogenetic role of glomerular CXCL13 expression in lupus nephritis. Clin Exp Immunol 2014;178:20-7.

13. Balaji S, Watson CL, Ranjan R, et al. Chemokine Involvement in Fetal and Adult Wound Healing. Adv Wound Care (New Rochelle) 2015;4:660-72.

14. Ding Y, Shen J, Zhang G, et al. CD40 controls CXCR5induced recruitment of myeloid-derived suppressor cells to gastric cancer. Oncotarget 2015;6:38901-11.

15. Sun Q, Chen L, Xu B, et al. Expression of chemokine receptor CXCR5 in gastric cancer and its clinical significance. Int J Clin Exp Pathol 2016;9:7202-8.

16. Chen L, Huang Z, Yao G, et al. The expression of CXCL13 and its relation to unfavorable clinical characteristics in young breast cancer. J Transl Med 2015;13:168. 
17. Razis E, Kalogeras KT, Kotoula V, et al. Improved outcome of high-risk early HER2 positive breast cancer with high CXCL13-CXCR5 messenger RNA expression. Clin Breast Cancer 2012;12:183-93.

18. Biswas S, Sengupta S, Roy Chowdhury S, et al. CXCL13CXCR5 co-expression regulates epithelial to mesenchymal transition of breast cancer cells during lymph node metastasis. Breast Cancer Res Treat 2014;143:265-76.

19. Mitkin NA, Hook CD, Schwartz AM, et al. p53-dependent expression of CXCR5 chemokine receptor in MCF-7 breast cancer cells. Sci Rep 2015;5:9330.

20. Qi XW, Xia SH, Yin Y, et al. Expression features of CXCR5 and its ligand, CXCL13 associated with poor prognosis of advanced colorectal cancer. Eur Rev Med Pharmacol Sci 2014;18:1916-24.

21. Zhu Z, Zhang X, Guo H, et al. CXCL13-CXCR5 axis promotes the growth and invasion of colon cancer cells via PI3K/AKT pathway. Mol Cell Biochem 2015;400:287-95.

22. Xing J, Zhang C, Yang X, et al. CXCR5+CD8+ T cells infiltrate the colorectal tumors and nearby lymph nodes, and are associated with enhanced IgG response in B cells. Exp Cell Res 2017;356:57-63.

23. Wang GZ, Cheng X, Zhou B, et al. The chemokine CXCL13 in lung cancers associated with environmental polycyclic aromatic hydrocarbons pollution. Elife 2015;4:e09419.

24. Singh R, Gupta P, Kloecker GH, et al. Expression and clinical significance of CXCR5/CXCL13 in human nonsmall cell lung carcinoma. Int J Oncol 2014;45:2232-40.

25. El-Haibi CP, Singh R, Gupta P, et al. Antibody Microarray Analysis of Signaling Networks Regulated by Cxcl13 and Cxcr5 in Prostate Cancer. J Proteomics Bioinform 2012;5:177-84.

26. Garg R, Blando JM, Perez CJ, et al. Protein Kinase C Epsilon Cooperates with PTEN Loss for Prostate Tumorigenesis through the CXCL13-CXCR5 Pathway. Cell Rep 2017;19:375-88.

27. Singh S, Singh R, Singh UP, et al. Clinical and biological significance of CXCR5 expressed by prostate cancer

Cite this article as: Zhu D, Ye W, Jiang J. Clinical significance of CXCL13/CXCR5 axis in human cancers. Transl Cancer Res 2018;7(6):1737-1742. doi: 10.21037/tcr.2018.11.26 specimens and cell lines. Int J Cancer 2009;125:2288-95.

28. Kim SJ, Ryu KJ, Hong M, et al. The serum CXCL13 level is associated with the Glasgow Prognostic Score in extranodal NK/T-cell lymphoma patients. J Hematol Oncol 2015;8:49.

29. Cha Z, Qian G, Zang Y, et al. Circulating CXCR5+CD4+ $T$ cells assist in the survival and growth of primary diffuse large B cell lymphoma cells through interleukin 10 pathway. Exp Cell Res 2017;350:154-60.

30. Wei Y, Lin C, Li H, et al. CXCL13 expression is prognostic and predictive for postoperative adjuvant chemotherapy benefit in patients with gastric cancer. Cancer Immunol Immunother 2018;67:261-9.

31. Razmkhah M, Jaberipour M, Safaei A, et al. Chemokine and chemokine receptors: a comparative study between metastatic and nonmetastatic lymph nodes in breast cancer patients. Eur Cytokine Netw 2012;23:72-7.

32. Xu L, Liang Z, Li S, et al. Signaling via the CXCR5/ ERK pathway is mediated by CXCL13 in mice with breast cancer. Oncol Lett 2018;15:9293-8.

33. El-Haibi CP, Singh R, Sharma PK, et al. CXCL13 mediates prostate cancer cell proliferation through JNK signalling and invasion through ERK activation. Cell Prolif 2011;44:311-9.

34. Sambandam Y, Sundaram K, Liu A, et al. CXCL13 activation of c-Myc induces RANK ligand expression in stromal/preosteoblast cells in the oral squamous cell carcinoma tumor-bone microenvironment. Oncogene 2013;32:97-105.

35. Duan Z, Gao J, Zhang L, et al. Phenotype and function of CXCR5+CD45RA-CD4+ T cells were altered in $\mathrm{HBV}$-related hepatocellular carcinoma and elevated serum CXCL13 predicted better prognosis. Oncotarget 2015;6:44239-53.

36. Del Grosso F, Coco S, Scaruffi P, et al. Role of CXCL13CXCR5 crosstalk between malignant neuroblastoma cells and Schwannian stromal cells in neuroblastic tumors. Mol Cancer Res 2011;9:815-23. 